\title{
Journal Club: High-dose methylprednisolone for acute traumatic spinal cord injury
}

\author{
A meta-analysis
}

Lisa J.W. Liu, BSc, MPH, Jan Rosner, MD, and Jacquelyn J. Cragg, MPH, PhD

Neurolog ${ }^{\circledR}$ 2020;95:272-274. doi:10.1212/WNL.0000000000009263

\author{
Correspondence \\ Dr. Cragg \\ Jacquelyn.cragg@icord.org
}

Methylprednisolone is a corticosteroid medication used in acute traumatic spinal cord injury (SCI) to tackle secondary injury cascades. Its use in acute SCI has been the subject of controversy for over 30 years. The second National Acute Spinal Cord Injury Study (NASCIS-2) demonstrated a small benefit of methylprednisolone, ${ }^{1}$ though this conclusion was derived from a post hoc subgroup analysis. ${ }^{2}$ Studies that followed did not reach the same conclusion and reported potential adverse effects of the drug. ${ }^{3}$ However, the 2017 AOSpine guideline continues to recommend high-dose methylprednisolone within 8 hours postinjury, ${ }^{4}$ based on a meta-analysis with rigid inclusion criteria. ${ }^{5}$ This Journal Club article reports on a study from Liu et al., ${ }^{6}$ who have attempted to resolve this controversy. The study provides an elegant example of meta-analytic methods and has important implications for neurologic clinical practice.

\section{Hypotheses and design}

Does acutely administered methylprednisolone improve neurologic recovery following traumatic SCI? To answer this important question, Liu et al. ${ }^{6}$ performed a meta-analysis of clinical trials and observational studies. The use of a meta-analysis is important here as it synthesizes all available evidence and provides the highest possible strength of recommendation for its use in clinical management.

\section{Methods}

This meta-analysis followed the Preferred Reporting Items for Systematic Reviews and MetaAnalyses (PRISMA) guidelines, which is an evidence-based minimum set of items for reporting in systematic reviews and meta-analyses. ${ }^{7}$ A comprehensive literature search was performed of PubMed and the Cochrane Library, and articles were further restricted to those published in journals included in the Science Citation Index. In addition to traditional database searches, manual searches of the reference lists of all of the relevant studies, review articles, and conference abstracts were also conducted. Searching multiple databases is recommended when conducting metaanalyses in order to identify all the relevant articles. For instance, the Cochrane Handbook suggests the use of MEDLINE (accessible through PubMed) and the Cochrane Central Register of Controlled Trials. ${ }^{8}$ Other commonly used databases include the Web of Science and Google Scholar. ${ }^{9}$

The review included studies in which participants were diagnosed with traumatic SCI. Methylprednisolone intervention was initiated within 8 hours after injury (based on prior evidence that patients treated after 8 hours postinjury recovered less motor function compared with placebo). ${ }^{10}$ Studies with a drug-naive control group were included. Other inclusion criteria for studies included reporting of neurologic outcomes, adverse events, or in-hospital costs. Two blinded reviewers evaluated articles identified in the initial search to reduce the risk of bias. From each article, the following data were then extracted: demographics, sample size, percent of patients with complete

From the Faculty of Pharmaceutical Sciences (L.J.W.L., J.R., J.J.C.) and International Collaboration on Repair Discoveries (ICORD) (L.J.W.L., J.J.C.), University of British Columbia, Vancouver, Canada; Spinal Cord Injury Center (J.R.), Balgrist University Hospital, University of Zurich; and Department of Neurology (J.R.), University Hospital Bern, Inselspital, University of Bern, Switzerland.

Go to Neurology.org/N for full disclosures. Funding information and disclosures deemed relevant by the authors, if any, are provided at the end of the article. 
injuries, percent of patients who received surgeries, length of follow-up, and outcomes (neurologic scores, adverse events, inhospital costs). For randomized trials, quality was assessed with a risk of bias assessment according to the Cochrane Handbook for Systematic Reviews and Interventions. ${ }^{8}$ This tool includes an assessment of various biases including selection bias, attrition bias, detection bias, performance bias, and reporting bias. For observational studies, quality was assessed using the NewcastleOttawa Scale (NOS). ${ }^{11}$ For the NOS, each study is judged on 3 broad categories: selection of study groups, comparability of study groups, and ascertainment of exposures and outcomes. The NOS ranges from 0 to 9 points; studies that score at least 6 are considered high quality (i.e., low risk of bias).

The outcomes were pooled using random-effects and fixedeffects meta-analyses. In a fixed-effects meta-analysis, we assume that each of the studies included are estimating the same treatment effect (true value). Therefore, the different effect estimates (from the different studies) are attributed purely to random sampling error. ${ }^{12}$ However, in most settings, this assumption does not hold: studies may have different inclusion criteria, treatments may have been administered by different protocols, and outcomes may have been captured differently. To account for these between-study differences (heterogeneity), randomeffects meta-analysis is used. ${ }^{12}$ Heterogeneity was assessed with $I^{2}$ statistics, which describe the percentage of variation across studies that is due to heterogeneity rather than chance, and a $95 \%$ confidence interval (CI) can be constructed for $I^{2} .8$

Continuous variables were summarized with pooled mean differences and 95\% CIs. Dichotomous variables were summarized with pooled risk ratios and 95\% CIs. In either case, the $95 \%$ CI can be interpreted as the certainty (confidence) that the true measure lies within the given range. ${ }^{8}$ Subgroup analyses were performed, one stratifying by study design (observational vs randomized trial) and by duration of follow-up ( $\leq 2$ months or $>2$ months).

\section{Results}

The broad electronic search strategy yielded 1,574 articles, and of these, 16 studies were included in the final analysis (3 randomized controlled trials and 13 observational studies). The quality assessment revealed that the observational studies and the randomized controlled trials were of modest or low risk of bias. The random-effects meta-analysis (graphically illustrated in the forest plot) did not detect a significant difference between the methylprednisolone and control groups for the pooled motor and sensory scores at last follow-up. This lack of difference was independent of study design and duration of follow-up. The corticosteroid was associated with a significantly higher incidence of adverse events, which included gastrointestinal hemorrhage and respiratory tract infection.

\section{Interpretation}

Liu et al. ${ }^{6}$ have brought to attention the use of methylprednisolone in acute traumatic SCI and concluded that high-dose methylprednisolone does not improve neurologic outcomes in acute traumatic SCI and may increase the risk of adverse events. They recommend against the use of this corticosteroid early after injury.

\section{Study strengths and limitations}

There are several strengths of this study and a few potential limitations related to its design, presentation of results, and research question. First, the authors formulated a focused research question, which can minimize heterogeneity between studies and aid in the identification and selection of studies.

Second, this is the largest meta-analysis of acute methylprednisolone to date, consisting of 3 clinical trials and 13 observational studies. This is particularly impressive given that SCI is considered an orphan condition. ${ }^{13}$ The included studies were restricted to English-language articles, though language-restricted meta-analyses tend to overestimate treatment effects by $2 \%$ on average. ${ }^{14}$

Third, articles were reviewed and data were extracted by 2 blinded reviewers, which reduces bias and reduces the likelihood of data entry errors.

Fourth, the results of the study were presented in accordance with PRISMA guidelines, ${ }^{7}$ including the use of clear forest plots (the graphical display of results from individual studies and the overall pooled result) and a clearly presented flow diagram of studies included in the final analysis.

Individual participant data, which were not used in this metaanalysis, have several advantages, including that overlapping sets of participants can be identified, and adjusted estimates can be produced where previously only unadjusted estimates were available. ${ }^{15}$ However, obtaining individual data is often not feasible as it requires contacting the authors of the original studies, and is particularly challenging for older studies.

With regards to the analysis, another major strength of the meta-analysis was the use of stratification by study design and duration of follow-up. Other potential stratifications could have included stratification by lesion level, particularly for adverse events (e.g., more respiratory complications in tetraplegia), and stratification by intention to treat vs per protocol. With regards to bias, the relationship between methylprednisolone use and neurologic outcomes could be confounded by other factors not included in the analyses, including surgical intervention, blood pressure maintenance, premorbid disability, and mechanism of injury. Confounding occurs when the true association between an exposure and an outcome is distorted by the presence of another variable (i.e., the confounder). ${ }^{16}$

Another strength of the Liu et al. ${ }^{6}$ meta-analysis was that an assessment of study quality was performed, although the quality score was not used in the meta-analysis. There are 
several techniques for incorporating quality scores in metaanalyses, including excluding studies of a certain quality, subgroup analyses by study quality, and incorporating quality scores as weights in the meta-analysis. ${ }^{17}$

Finally, the meta-analysis included studies with a range of ages and both complete and incomplete injuries, which increases the generalizability of this study.

Overall, despite the study limitations, this study is relevant to neurologic practice. Indeed, this study is the most comprehensive synthesis of the relevant literature to date. This in combination with the other major strengths of this study make it an important contribution to neurologic practice.

\section{Study funding}

No targeted funding reported.

\section{Disclosure}

The authors report no disclosures relevant to the manuscript. Go to Neurology.org/N for full disclosures.

\section{Appendix Authors}

\begin{tabular}{lll}
\hline Name & Location & Contribution \\
\hline $\begin{array}{l}\text { Lisa J.W. Liu, } \\
\text { BSc, MPH }\end{array}$ & $\begin{array}{l}\text { University of } \\
\text { British Columbia, } \\
\text { Canada }\end{array}$ & $\begin{array}{l}\text { Interpreting the results of the } \\
\text { study (critical appraisal) and } \\
\text { developing the manuscript }\end{array}$ \\
\hline $\begin{array}{l}\text { Jan Rosner, } \\
\text { MD }\end{array}$ & $\begin{array}{l}\text { University of } \\
\text { Zurich, } \\
\text { Switzerland }\end{array}$ & $\begin{array}{l}\text { Interpreting the results of the } \\
\text { study (critical appraisal) and } \\
\text { developing the manuscript }\end{array}$ \\
\hline $\begin{array}{l}\text { Jacquelyn J. } \\
\text { Cragg, MPH, } \\
\text { PhD }\end{array}$ & $\begin{array}{l}\text { University of } \\
\text { British Columbia, } \\
\text { Canada }\end{array}$ & $\begin{array}{l}\text { Interpreting the results of the } \\
\text { study (critical appraisal) and } \\
\text { developing the manuscript }\end{array}$ \\
\hline
\end{tabular}

\section{References}

1. Bracken MB, Shepard MJ, Collins WF, et al. A randomized, controlled trial of methylprednisolone or naloxone in the treatment of acute spinal-cord injury: results of the Second National Acute Spinal Cord Injury Study. N Engl J Med 1990;322: $1405-1411$.

2. Boody BS, Savage JW, Eck JC, Hodges SD. Steroid use in adult patients with incomplete spinal cord injuries. Clin Spine Surg 2016;29:92-94.

3. Evaniew N, Noonan VK, Fallah N, et al. Methylprednisolone for the treatment of patients with acute spinal cord injuries: a propensity score-matched cohort study from a Canadian multi-center spinal cord injury registry. J Neurotrauma 2015;32: 1674-1683.

4. Fehlings MG, Wilson JR, Tetreault LA, et al. A clinical practice guideline for the management of patients with acute spinal cord injury: recommendation on the use of methylprednisolone sodium succinate. Glob Spine J 2017;7: 203s-211s.

5. Fehlings MG, Wilson JR, Harrop JS, et al. Efficacy and safety of methylprednisolone sodium succinate in acute spinal cord injury: a systematic review. Glob Spine J 2017; 7: $116 \mathrm{~s}-137 \mathrm{~s}$.

6. Liu Z, Yang Y, He L, et al. High-dose methylprednisolone for acute traumatic spinal cord injury: a meta-analysis. Neurology 2019;93:e841-e850.

7. Moher D, Liberati A, Tetzlaff J, Altman DG. Preferred reporting items for systematic reviews and meta-analyses: the PRISMA statement. Int J Surg 2010;8 336-341.

8. Higgins JPT, Green S, eds. Cochrane handbook for systematic reviews of interventions version 5.1.0 [online]. Available at: handbook-5-1.cochrane.org/. Accessed December 10, 2019.

9. Bramer WM, Rethlefsen ML, Kleijnen J, Franco OH. Optimal database combinations for literature searches in systematic reviews: a prospective exploratory study. Syst Rev $2017 ; 6: 245$.

10. Bracken MB, Shepard MJ, Collins WF Jr, et al. Methylprednisolone or naloxone treatment after acute spinal cord injury: 1-year follow-up data: results of the second National Acute Spinal Cord Injury Study. J Neurosurg 1992;76:23-31.

11. Stang A. Critical evaluation of the Newcastle-Ottawa Scale for the assessment of the quality of nonrandomized studies in meta-analyses. Eur J Epidemiol 2010;25: 603-605.

12. Tufanaru C, Munn Z, Stephenson M, Aromataris E. Fixed or random effects metaanalysis? Common methodological issues in systematic reviews of effectiveness. Int J Evid Based Healthc 2015;13:196-207.

13. Burke KA, Freeman SN, Imoisili MA, Coté TR. The impact of the orphan drug act on the development and advancement of neurological products for rare diseases: a descriptive review. Clin Pharmacol Ther 2010;88:449-453.

14. Moher D, Cook DJ, Eastwood S, Olkin I, Rennie D, Stroup DF. Improving the quality of reports of meta-analyses of randomised controlled trials: the QUOROM statement. Lancet 1999;354:1896-1900.

15. Riley RD, Lambert PC, Abo-Zaid G. Meta-analysis of individual participant data: rationale, conduct, and reporting. BMJ 2010;340:521-525.

16. VanderWeele TJ, Shpitser I. On the definition of a confounder. Ann Stat 2013;41: 196-220.

17. Ahn S, Becker BJ. Incorporating quality scores in meta-analysis. J Educ Behav Stat 2011;36:555-585.

\section{COVID-19 and Neurologic Disease: Call for Papers!}

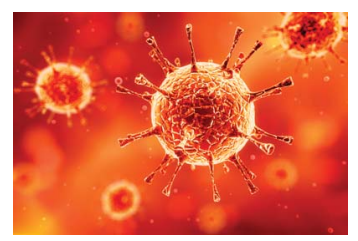

The editors of Neurology are interested in papers that address the neurological aspects of COVID-19 infection and challenges to the management of patients with chronic neurological conditions who have, or are at risk for, the infection. Relevant papers that pass initial internal review will undergo expedited peer review and online publication. We will consider papers posted in preprint servers.

Submit observational studies and clinical trials as Articles and case series and case reports under the Clinical/Scientific Notes category to https://submit.neurology.org/ today! 


\section{Neurology}

\section{Journal Club: High-dose methylprednisolone for acute traumatic spinal cord injury: A meta-analysis}

Lisa J.W. Liu, Jan Rosner and Jacquelyn J. Cragg

Neurology 2020;95;272-274 Published Online before print April 8, 2020

DOI 10.1212/WNL.0000000000009263

\section{This information is current as of April 8, 2020}

\section{Updated Information \&} Services

References

Subspecialty Collections

Permissions \& Licensing

Reprints including high resolution figures, can be found at: http://n.neurology.org/content/95/6/272.full

This article cites 16 articles, 1 of which you can access for free at: http://n.neurology.org/content/95/6/272.full\#ref-list-1

This article, along with others on similar topics, appears in the following collection(s):

\section{All Spinal Cord}

http://n.neurology.org/cgi/collection/all_spinal_cord Spinal cord trauma

http://n.neurology.org/cgi/collection/spinal_cord_trauma

Spinal cord trauma; see Trauma/spinal cord trauma

$\mathrm{http}: / /$ n.neurology.org/cgi/collection/spinal_cord_trauma-see_trauma-s pinal_cord_trauma

Information about reproducing this article in parts (figures,tables) or in its entirety can be found online at:

http://www.neurology.org/about/about_the_journal\#permissions

Information about ordering reprints can be found online:

http://n.neurology.org/subscribers/advertise

Neurology ${ }^{\circledR}$ is the official journal of the American Academy of Neurology. Published continuously since 1951, it is now a weekly with 48 issues per year. Copyright () 2020 American Academy of Neurology. All rights reserved. Print ISSN: 0028-3878. Online ISSN: 1526-632X.

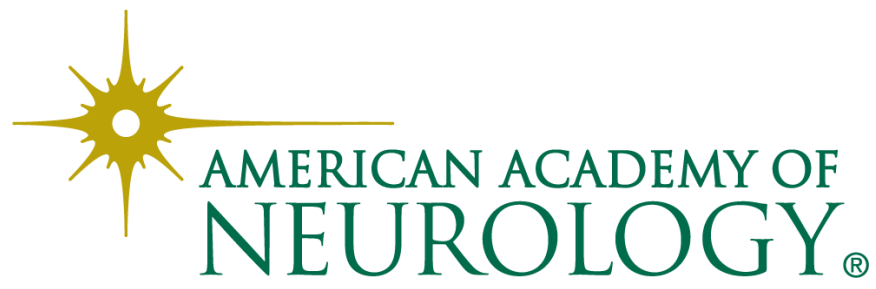

